\title{
Zinc intake and serum levels in Portuguese women living in Lisbon area
}

\section{Niveis séricos e ingestão de zinco num grupo de meia-idade de mulheres Portuguesas que vivem na área de Lisboa}

\author{
Nelson R. Tavares ${ }^{1}$, Abreu S ${ }^{2}$, S. Costa ${ }^{3}$, A. Miranda ${ }^{3}$, Catarina Rosado ${ }^{1}$ \\ ${ }^{1}$ CBIOS - Universidade Lusófona's Research Center for Biosciences and Health Technologies (UDE), Campo Grande 376, \\ 1749-024, Lisboa, Portugal \\ ${ }^{2}$ CIDAF, Faculdade de Desporto, Universidade do Porto, Rua Doutor Plácido da Costa 91, 4200-400 Porto, Portugal. \\ ${ }^{3}$ DPS, Instituto Nacional de Saúde Doutor Ricardo Jorge, Avenida Padre Cruz, 1649-016 Lisboa, Portugal. \\ Email: nelson.tavares@ulusofona.pt
}

\begin{abstract}
Zinc is an essential micronutrient, especially in relation to its impact on immune function, bone mass, cognitive function and oxidative stress. Inadequate intakes of micronutrients such as zinc negatively affect nutritional status. Data is limited on zinc intake and serum levels in Portuguese women. In this study, the intake and serum levels of zinc in a group $(\mathrm{n}=17)$ of middle-aged women living in Lisbon area were assessed. Dietary intakes were collected using the four-day recall-record method. Serum levels of zinc were obtained from blood samples. In relation to the zinc Dietary Reference Intakes, zinc intakes were inadequate and serum zinc results showed deficiency in this group.
\end{abstract}

Keywords: zinc, intake, serum levels, women

\section{Resumo}

O zinco é um micronutriente essencial, especialmente em relação ao seu impacto na função imunológica, massa óssea, função cognitiva e stresse oxidativo. A ingestão inadequada de micronutrientes pode afetar negativamente o estado nutricional. São escassos os dados relativos à ingestão e aos níveis plasmáticos de zinco em mulheres portuguesas. Neste estudo, foram avaliados os níveis plasmáticos e a ingestão em 17 mulheres de meia-idade que vivem na área de Lisboa. A ingestão alimentar foi determinada a partir de 4 recordatórios alimentares. Para a obtenção dos níveis séricos de zinco, foram colhidas amostras de sangue. Em relação à Dietary Reference Intake de zinco, a ingestão de zinco mostra inadequação; e os níveis séricos deficiência em zinco. Os resultados obtidos mostram uma deficiência de zinco neste grupo de mulheres.

Palavras-chave: zinco, ingestão, níveis séricos, mulher 


\section{Introduction}

Zinc is an essential micronutrient for human metabolism that catalyzes more than 100 enzymes, facilitates protein folding, and helps regulate gene expression ${ }^{[1]}$. Studies in Latin American countries and the U.S. showed that the average zinc intake varies between $50 \%$ and $80 \%$ of the recommendation, regardless of age, gender and race ${ }^{[2]}$. Inadequate zinc intakes as well as alterations of zinc serum levels have been especially demonstrated in hospitalised people ${ }^{[3]}$. However, data on zinc intake and serum levels in middle-aged people is very limit$\mathrm{ed}^{\left[{ }^{[4]}\right.}$. A number of zinc-related behavorial abnormalities have also been described in humans, including changes in mood, loss of affect and emotional labiality, irritability and depression ${ }^{[5]}$. Aging contributes to increase the risk of deficiency as a result of multiple physiological, social, psychological and economic factors ${ }^{[6]}$. Providing information about dietary habits of typical middle-age women living in Lisbon area, and the possible consequences on the incidence of zinc deficiency in this group, would be useful for the determination of specific needs in relation to zinc in such a population. Therefore, the aim of this work was to describe zinc intakes and status in a middle-aged women group living in Lisbon area.

\section{Materials and Methods}

Women aged between 40 years and 65 years were recruited among Universidade Lusófona employees. The inclusion criteria were healthy subjects aged between 40 years and 65 years, non-pregnant, non-lactating and not taking zinc supplements. Participants were informed of the purpose and risk of the protocol. Eighteen women volunteered after interview and were recruited in accordance with the Helsinki Declaration of $2008^{[7]}$.

For zinc determination, blood was collected in Vacutain$\mathrm{er}^{\circledR}$ tubes. Samples were kept on ice immediately after drawing, and then centrifuged at $1000 \mathrm{~g}$ for 15 minutes at $4{ }^{\circ} \mathrm{C}$. Serum fractions containing zinc were immediately isolated, aliquoted and stored at $-20{ }^{\circ} \mathrm{C}$. Zinc levels were determined by flame atomic absorption spectrometry. Two study subjects refused to give a blood sample and one sample in the group was refused due to hemolysis.

Dietary intake was measured via a four-day dietary record that included three weekdays and one weekend day. Portion sizes of foods and beverages consumed were estimated using household measures (cups, glasses, spoons, slices, etc.) and other props (including food wrappers or containers) as an aid in determining serving sizes. The nutrient analysis was performed using the software Food Processor SQL (ESHA Research Inc., Salem, OR, USA). This program uses nutritional information from the United States that has been adapted for use with typical Portuguese foods and beverages. Nutrient means of the four days of diet record were used in the analysis. The food record is often used as the gold standard for

\section{Introdução}

O zinco é um micronutriente essencial para o metabolismo humano, catalisa mais de cem enzimas, facilita o enovelamento das proteínas e ajuda a regular a expressão dos genes ${ }^{[1}$ ]. Estudos realizados em países da América Latina e nos EUA mostram que a ingestão média de zinco varia entre $50 \%$ e 80 $\%$ do recomendado, independentemente da idade, sexo e raça [2]. A ingestão inadequada de zinco, bem como nos níveis deficientes de zinco estão bem demonstrados em pessoas hospitalizadas ${ }^{[3]}$. No entanto, os dados sobre a ingestão de zinco e os seus níveis em pessoas não institucionalizadas de meia-idade são escassos ${ }^{[4]}$. Foram descritas algumas alterações comportamentais relacionadas com o zinco em seres humanos. Estas incluem alterações no humor, perda de afeto e equilíbrio emocional, irritabilidade e depressão ${ }^{[5]}$. O envelhecimento contribui para aumentar o risco de deficiência como resultado de múltiplos fatores fisiológicos, sociais, psicológicos e económicos ${ }^{[6]}$. Obter informações sobre os hábitos alimentares de mulheres de meia-idade não institucionalizadas que vivem na área de Lisboa e as possíveis consequências sobre a incidência de deficiência de zinco neste grupo será útil para a determinação de necessidades específicas em relação ao zinco. $\mathrm{O}$ objetivo deste trabalho foi descrever a ingestão e os níveis de zinco neste grupo de mulheres de meia-idade que vivem na área de Lisboa.

\section{Materiais e Métodos}

Mulheres com idade entre os 40 anos e os 65 anos foram recrutadas entre os funcionários da Universidade Lusófona. Os critérios de inclusão foram: indivíduos saudáveis com idades entre os 40 anos e os 65 anos, que não estivessem grávidas ou a amamentar, e não tomar suplementos alimentares contendo zinco. Os participantes foram informados sobre a finalidade e risco do protocolo. Após entrevista foram recrutadas dezoito mulheres de acordo com a Declaração de Helsinki de $2008^{[7]}$. Para a determinação de zinco, o sangue foi colhido em tubos Vacutainer ${ }^{\circledR}$. As amostras foram mantidas em gelo após a colheita, e, em seguida, centrifugadas a $1000 \mathrm{~g}$ durante 15 $\min$ a $4{ }^{\circ} \mathrm{C}$. O soro para a determinação do zinco foi isolado, aliquotado e armazenado a $-20^{\circ} \mathrm{C}$. A análise do ião zinco foi realizada num espectrofotómetro de absorção atómica equipado com atomizador em chama e uma lâmpada de cátodo oco para zinco.

A ingestão alimentar foi avaliada através de registo alimentar de 4 dias, que incluiu três dias da semana e um dia de fim-desemana. O tamanho das porções dos alimentos e bebidas consumidos foram estimadas usando medidas caseiras (chávenas, copos, colheres, fatias, etc) e outros (copos, embalagens de alimentos ou recipientes) como uma ajuda na determinação de tamanhos de porção. A análise de nutrientes foi realizada utilizando o software SQL Processador de Alimentos ( ESHA Research Inc. , Salem, OR , EUA). Este programa usa informações nutricionais dos Estados Unidos, que foram adaptadas para a utilização de alimentos típicos portugueses e bebidas. 
characterization of current and usual diet in groups or individuals ${ }^{[8,9]}$.

All statistical analyses were carried out with SPSS 15.0, with data presentation as mean \pm standard deviation (SD). A t test was conducted for significant values at $\mathrm{p}<0.05$.

\section{Results}

Seventeen subjects completed the study. All were Caucasian women with a mean age 52.3 years ( $\mathrm{SD}=7.3$ years). Zinc intake is shown in Table 1. In this group of middle-aged women, the percentage of subjects who met the European Recommended Daily Allowances (RDA) ${ }^{[10]}$ was $53 \%$. The estimated average daily zinc intake was $6.76 \mathrm{mg} / \mathrm{d}$. Serum zinc levels were determined for fourteen subjects. The percentage of subjects presenting a serum zinc concentration above $10.7 \mu \mathrm{mol} / \mathrm{L}$, usually considered as the cut off level for zinc deficiency ${ }^{[11]}$, was $43 \%$ (Table 2 ). The average serum zinc concentration was $10.42 \mu \mathrm{mol} / \mathrm{L}(\mathrm{SD}=1.93 \mu \mathrm{mol} / \mathrm{L})$. The relationships between indices of zinc nutritive status are shown in Table 3. Zinc intake was not significantly correlated with zinc serum concentration.
$\mathrm{Na}$ análise, foram utilizados médias dos nutrientes de 4 dias do registo alimentar. $\mathrm{O}$ registo de alimentos é frequentemente usado como o padrão-ouro para a caracterização da dieta atual e comum em grupos ou indivíduos ${ }^{[8,9]}$.

Todas as análises estatísticas foram realizadas com SPSS 15.0 com apresentação de dados como média \pm desvio padrão (DP). $\mathrm{O}$ teste $\mathrm{t}$ foi aplicado e considerado significativo quando $\mathrm{p}<0.05$.

\section{Resultados}

Dezassete participantes completaram o estudo. Todas mulheres caucasianas, com uma média de idade de 52,3 anos (DP $=7,3$ anos). Os valores referentes à ingestão de zinco são mostrados na Tabela 1 . Neste grupo de mulheres de meiaidade, a percentagem de indivíduos que ingeriu zinco de acordo com as Doses Diárias Recomendadas (DDR) ${ }^{[10]}$ foi de 53 $\%$. A ingestão média diária estimada de zinco foi de $6,76 \mathrm{mg} /$ dia (Tabela 1). Apenas foram consideradas catorze alíquotas de soro para a determinação de zinco. A percentagem de indivíduos que apresentaram uma concentração de zinco no soro acima de $10,7 \mu \mathrm{mol} / \mathrm{L}$, geralmente considerado como o ponto de corte para a deficiência de zinco ${ }^{[11]}$, foi de $43 \%$ (Tabela 2 ). A concentração média de zinco no soro foi de $10,42 \mu \mathrm{mol} / \mathrm{L}$ $(\mathrm{DP}=1,93 \mu \mathrm{mol} / \mathrm{L})$. A correlação entre os índices do estado nutritivo de zinco são mostrados na Tabela 3. Os valores de ingestão de zinco não estão significativamente correlacionados com a concentração de zinco sérico.

Table 1/ Tabela 1 - Zinc intake (mean $\pm S D$ )

Ingestão de zinco (média $\pm D P$ )

\begin{tabular}{|c|c|}
\multicolumn{1}{c}{$n=17$} \\
\hline Energy (MJ/d) & \\
Energia (MJ/d) & $5.45 \pm 7.34$ \\
Zinc (mg/d) & \\
\hline Zinco (mg/d) $(\mathrm{mg} / \mathrm{MJ})$ & \\
Zinco (mg/MJ) & $1.24 \pm 0.40 \pm 2.09$ \\
\hline $\begin{array}{c}\text { Percentage of subjects } \\
\text { with zinc intake lower } \\
\text { than RDA } \\
\text { Percentagem de } \\
\text { indivíduos com }\end{array}$ \\
$\begin{array}{l}\text { ingestão de zinco } \\
\text { menor que a Dose } \\
\text { Diária Recomendada }\end{array}$ \\
\hline
\end{tabular}


Table 2/ Tabela 2 - Zinc serum levels (mean $\pm S D$ )

Níveis séricos de zinco $($ mean $\pm S D)$

\begin{tabular}{|c|c|}
\hline Serum zinc $(\mu \mathrm{mol} / \mathrm{L})$ & \\
Zinco sérico $(\mu \mathrm{mol} / \mathrm{L})$ & $10.42 \pm 1.93$ \\
\hline Minimum serum zinc & \\
Zinco sérico mínimo & \\
( $\mu$ mol/L) & \\
Percentage of subjects & \\
with smaller than & \\
minimum serum zinc & \\
Percentagem de & \\
indivíduos com zinco & \\
sérico menor que o & \\
\hline
\end{tabular}

\section{Discussion}

The zinc intakes and status in a middle-aged women group living in Lisbon area are low. The zinc density in this group of women was $5.2 \mathrm{mg} /(1000 \mathrm{kcal})$, below the American adult's diet which is approximately $5.6 \mathrm{mg} /$ $(1000 \mathrm{kcal}){ }^{[12]}$. The average serum zinc concentration of the women in this study was lower than $10.7 \mu \mathrm{mol} / \mathrm{L}$ and only $43 \%$ of participants were classified as nonzinc deficient. These serum zinc concentrations were lower than the $13.01 \mu \mathrm{mol} / \mathrm{L}$ reported for a middle-aged group in the ZENITH study ${ }^{[4]}$. The apparent low zinc status suggests that dietary patterns might compromise dietary zinc bioavailability in our population. Some blood samples were drawn as non-fasting in the morning, others in the afternoon. The time of the day that blood samples were drawn could have a significant effect on serum zinc concentrations, such that serum zinc could be higher in the morning than in the afternoon samples ${ }^{[13] .}$ On the other hand, blood sample collection hemolysis and delays in the separation of serum could

\section{Discussão}

Os níveis séricos e de ingestão de zinco neste grupo de mulheres de meia-idade que vivem na zona de Lisboa são baixos. O teor em zinco neste grupo de mulheres foi de 5,2 $\mathrm{mg} /(1000 \mathrm{kcal})$, inferior aos valores encontrados na dieta do adulto nos EUA, que é de aproximadamente $5,6 \mathrm{mg} /(1000 \mathrm{kcal}){ }^{[12]}$.A concentração média de zinco no soro das mulheres neste estudo é inferior a $10,7 \mathrm{mmol} / \mathrm{L}$ e apenas $43 \%$ das participantes foram classificadas como não apresentando deficiência nos níveis de zinco. Sendo essas concentrações menores que 13,01 mmol / L, valor encontrado para um grupo de meia-idade no estudo ZENITH ${ }^{[4]}$. As razões para o aparente baixo nível de zinco sugerem que os padrões alimentares podem comprometer a biodisponibilidade do zinco na dieta da nossa população. Algumas amostras de sangue foram colhidas de manhã sem ter sido observado o jejum e outras na parte da tarde. A altura do dia em que as amostras de sangue foram colhidas pode ter um efeito significativo sobre as concentrações de zinco 
increase serum zinc levels. Serum zinc concentrations have been reported to be lowest in young children, increasing steadily with age, peaking between 18 years and 25 years of age, decreasing slowly during adulthood, and dropping off after 65 years -70 years of age [13]. The effects of other potentially confounding variables on serum zinc concentrations, such as low serum albumin, high white blood cell count or current use of oral contraceptives ${ }^{[13]}$, were not studied.

It was also of interest to note that there are no discrepancies between dietary and biochemical estimates of risk for low zinc status in our population.

Recommendations on zinc supplementation and good food sources of this mineral could be made for women with zinc deficiency in this group. Red meat, whole wheat, raisins, unrefined cereals (high content, low bioavailability) and fortified cereals would be considered adequate. Milk, fruit and vegetables are low in zinc ${ }^{[14]}$. Increased dietary zinc intake would not cause harm if the $25 \mathrm{mg} / \mathrm{d}$ Tolerable Upper Intake Level was respected ${ }^{[15]}$.

\section{Conclusion}

Zinc serum levels and dietary intake results may suggest that the zinc status of this group of Lisbon area middle-aged women was low. This is of concern for this population, given the requirement for zinc for optimal immune function and importance for human metabolism. Consequently, these results should be useful for further studies on zinc intake and serum levels for middle-aged women.

\section{Conflict of Interests}

The authors declare that there are no financial and personal relationships that could be viewed as a potential conflict of interests. no soro, visto que podem ser mais elevadas de manhã do que nas amostras da tarde ${ }^{[13]}$.

Por outro lado, a hemólise resultante da colheita e atrasos na separação do soro podem provocar o aumento dos níveis séricos de zinco. Apenas uma amostra, neste grupo, foi recusado devido a hemólise. Duas participantes recusaram ceder amostra de sangue. Concentrações de zinco no soro são menores em crianças pequenas, aumentam progressivamente com a idade, atingindo um pico entre os 18 anos e os 25 anos, diminuindo lentamente durante a vida adulta, e abruptamente após os 65 anos-70 anos de idade ${ }^{[13]}$. O efeito de outras variáveis potencialmente perturbadoras das concentrações de zinco no soro como níveis baixos de albumina sérica, níveis elevados de glóbulos brancos ou a utilização de anticoncepcionais orais ${ }^{[13]}$, não foram estudados. Também foi interessante verificar que não existe discrepância entre as estimativas da ingestão dietética e os níveis sérios de zinco na nossa população. Recomendações sobre a suplementação de zinco e acesso a boas fontes alimentares deste mineral podem ser feitas às mulheres que apresentaram deficiência de zinco neste grupo. A carne vermelha, trigo integral, passas, cereais não refinados (alto teor, baixa biodisponibilidade) e cereais fortificados seriam considerados adequados. Leite, frutas e legumes são pobres em zinco ${ }^{[14]}$. O aumento da ingestão dietética de zinco não será um problema se o nível Máximo de Ingestão Tolerável de $25 \mathrm{mg} /$ dia respeitado ${ }^{[15]}$.

\section{Conclusão}

Os resultados obtidos quanto aos níveis sérios e a ingestão de zinco podem sugerir que neste grupo de mulheres de meia-idade da área de Lisboa, são baixos. Sendo motivo de preocupação para esta população, dada a necessidade de zinco para uma óptima função imunitária e importância que tem para o metabolismo humano. Consequentemente, esses resultados devem ser úteis para novos estudos sobre a avaliação dos níveis sérios e de ingestão de zinco para as mulheres de meiaidade.

\section{Conflito de Interesses}

Os autores declaram não existir qualquer relação de natureza financeira ou pessoal que possa, ser entendida como, ou representar, um potencial conflito de interesses. 


\section{References / Referências}

1. Saper RB, Rash R. Zinc: an essential micronutrient. Am Fam Physician 2009; 79:76872.

2. Salgueiro MJ, Bioch MZ, Lysionek A, Sarabia MI, Caro R, Paoli TD, et al. Zinc as an essential micronutrient: a review. Nutr Res. 2000; 20: 737-55.

3. Schmuck A, Roussel AM, Arnaud J, Ducros V, Favier A \& Franco A. Analyzed dietary intakes, plasma concentrations of zinc, copper, and selenium, and related antioxidant enzyme activities in hospitalized elderly women. J. Am. Coll. Nutr 1996; 15:462-468.

4. Andriollo-Sanchez M, Hininger-Favier I, Meunier N, Toti E, Zaccaria M, BrandoliniBunlon $\mathrm{M}$, et al.Zinc intake and status in middle-aged and older European subjects: the ZENITH study. Eur J Clin Nutr 2005; 59 Suppl 2: S37-41.

5. Brown H, Wuehler E, Peerson M. The importance of $\mathrm{Zn}$ in human nutrition and estimation of the global prevalence of zinc deficiency. Food \& Nutrition Bulletin, 2001;22:113-125
6. Drewnowski A, Shulz JM. Impact of aging on eating behaviours, food choices, nutrition, and health status. J Nutr Health Aging 2001;5:75-79.

7. Worl Medical Association Declaration of Helsinki. Seoul 2008. In URL: http://www. wma.net/e/policy/b3.htm (accessed 26 january 2013).

8. Barreti-Connor E. Nutrition epidemiology: how do we know what they ate?. Am J Clin Nutr 1991; 54:182S-7S.

9. Rutishause I. Dietary intake measurements. Public Health Nutrition 2005: 8(7A), 11001107.

10. Commission Directive 2008/100/EC of 28 October 2008 amending Council Directive 90/496/EEC on nutrition labelling for foodstuffs as regards recommended daily allowances, energy conversion factors and definitions. http://eur-lex.europa.eu/LexUriServ/ LexUriServ.do?uri=OJ:L:2008:285:0009:00 12:EN:PDF (English) (accessed 19 October 2014).
11. Burtis C, Tietz E. Textbook of Clinical Chemistry, Third Edition. Ashwood, eds. Philadelphia, PA: WB Saunders, 1998

12. Mahan L, Escott-Stump S, Raymond J. Krause's Food \& The Nutrition Care Process. 13th ed. ISBN 978-1-4377-2233-8.

13. Hotz C, Peerson J, Brown K. Suggested lower cutoffs of serum zinc concentrations for assessing zinc status: reanalysis of the second National Health and Nutrition Examination Survey data (1976-1980). Am J Clin Nutr October 2003; 78:756-64.

14. Sandstead H, Smith Jr S. Deliberations and evaluations of approaches, endpoints and paradigms for determining zinc dietary recommendations. J Nutr 1996; 126: 2410s2418 s.

15. SCF. 2003. Opinion of the Scientific Committee on Food on tolerable upper intake leve of zinc (SCF/CS/NUT/UPPLEV/62 Final), http://ec.europa.eu/food/fs/sc/scf/out177 en.pdf (accessed 19th October 2014) 